\title{
SENSORIAMENTO REMOTO APLICADO AO MAPEAMENTO E QUANTIFICAÇÃO DE ÁREAS DE MANGUEZAL NO ESTADO DE SERGIPE
}

Bruno Barros de Souza

Mestre em Ecologia e Conservação pela Universidade Federal de Sergipe brunobarros.eco@gmail.com

José Damião de Melo

Mestre em Modelagem Computacional de Conhecimento pela Universidade Federal de Alagoas damiao@damiaomelo.com.br

$\begin{array}{r}\begin{array}{r}\text { Monica Correa } \\ \text { Mestre em Geociências pela Universidade de São Paulo } \\ \text { monica nica@hotmail.com }\end{array} \\ \hline\end{array}$

Aline Oliveira moura Bacharel em Engenharia Florestal pela Universidade Federal de Sergipe ninefloresta@yahoo.com.br

Francisco Freitas Santos Mestre em Agroecossistemas pela Universidade Federal de Sergipe franciscofreitas.santos@adema.se.gov.br

Maria Cleusa Guimarães Mestre em Fitotecnia pela Universidade Federal de Viçosa mcleusag@yahoo.com.br

Halesio Milton Correia de Barros Neto Bacharel em Ciências Biológicas pela Universidade Federal de Sergipe halesio.barros388@gmail.com

\section{RESUMO}

Apesar de sua importância ambiental, principalmente na manutenção de diversos serviços ecossistêmicos, os manguezais são alvo de uma intensa redução de suas áreas. Estima-se que 1 a $2 \%$ de seus remanescentes em todo 0 mundo desapareçam a cada ano, o que torna urgente o seu mapeamento e o delineamento de políticas públicas para sua preservação. Apesar da histórica participação do poder público neste processo de degradação, o mapeamento e quantificação das áreas de manguezal tornou-se um objetivo estratégico para a criação e implementação de ações para sua conservação no estado de Sergipe. Este mapeamento foi realizado através de técnicas de classificação supervisionada de imagens RapidEye das áreas de interesse, e resultaram no mapeamento de $251,0786 \mathrm{Km}^{2}$ de manguezais distribuídos em 16 municípios da costa sergipana. Este valor equivale a cerca de $1,14 \%$ do território do Estado. As informações aqui geradas foram compiladas em mapas e arquivos do tipo shape, disponibilizadas ao público através de meios digitais, como dvd's temáticos e internet, de forma gratuita, com a finalidade subsidiar iniciativas voltadas à ampliação do conhecimento sobre o tema e a melhor gestão ambiental dos remanescentes mapeados.

Palavras-chave: Manguezais; Geoprocessamento; Gestão Ambiental.

\section{REMOTE SENSING APPLIED TO MANGROVE MAPPING AND QUANTIFICATION IN SERGIPE STATE}

\section{ABSTRACT}

Beside of the environmental importance, particularly in the maintenance of diverse ecosystem services, mangroves are the subject of an intense reduction of their

Recebido em 08/12/2014

Aprovado para publicação em 15/01/2016 
areas. It is estimated that $1-2 \%$ of the remaining worldwide area are disappearing each year, making urgent their mapping and the design of public policies for their preservation. Despite the historical participation of the government in this process of degradation, the mapping and quantification of mangrove areas has become a strategic objective for the creation and application of measures for their conservation in the state of Sergipe. This mapping was developed by using techniques of supervised classification by using RapidEye images of areas of interest and resulted in the mapping of $251.0786 \mathrm{Km}^{2}$ of mangroves distributed in 16 municipalities of Sergipe coast. This amount is equivalent to about $1.14 \%$ of the state territory. The information generated here were compiled into shape maps and files, available to the public through digital media such as thematic DVDs and internet, for free, in order to encourage initiatives intended to broaden the knowledge on the subject and the best environmental management about the remaining area that was mapped.

Keywords: Mangrove; Geoprocessing; Environmental Management.

\section{INTRODUÇÃO}

Os manguezais são ecossistemas tropicais severamente degradados em todo o mundo. Estimativas apontam que anualmente perde-se $1 \%$ a $2 \%$ de sua cobertura total, e desde 1980, cerca de $25 \%$ de sua área foi suprimida (DUKE et al., 2007; WILKIE e FORTUNA, 2003). As principais causas deste processo são as ações humanas, dentre elas: a expansão das áreas urbanas; a poluição do solo e dos recursos hídricos; e a instalação de empreendimentos de aquicultura (ALONGI, 2002; DUKE et al., 2007; VALIELA et al., 2001). Além destas, as mudanças no clima e consequente elevação no nível dos oceanos têm gerado grande pressão sobre estes ambientes (GILMAN et al., 2006).

Uma das consequências desta degradação é o comprometimento de uma série de serviços ecossistêmicos que dependem direta ou indiretamente dos manguezais, como: a diversidade da vida marinha; a subsistência de comunidades tradicionais; a qualidade das águas; e o armazenamento e transferência de carbono para o ambiente aquático (GILMAN et al., 2006). Assim, considerando seu papel nos setores econômico, ecológico e social, a preservação dos seus remanescentes assume caráter de grande importância (DUKE et al., 2007).

Pode-se afirmar que a preservação deste ecossistema é fundamental para a manutenção da qualidade de vida das comunidades litorâneas, e que a criação de mecanismos legais voltados à sua proteção é importante para a sua efetiva conservação (RÖNNBÄCK, 1999).

Neste sentido, em 1965 o código florestal brasileiro definiu os manguezais como Áreas de Preservação Permanente (APP), passando a ser vedado seu uso ou supressão exceto nos casos previstos em Lei e apenas quando autorizados pelo órgão ambiental competente (BRASIL, 1965; BRASIL, 2006).

Apesar de ter representado um avanço na conservação ambiental, a criação de leis restritivas quanto à ocupação e exploração destas áreas não foi suficiente para impedir 0 avanço de sua degradação. A urbanização acelerada e a implantação de empreendimentos em regiões costeiras, em muitos casos incentivados pelo Estado, continuam a causar grandes impactos aos manguezais.

Nos estados de São Paulo (RODRIGUES et al., 1999); Pernambuco (GUIMARÃES et al., 2009), Bahia (FIDELMAN et al., 2000; NOGUEIRA e DOMINGUEZ, 2007), e Sergipe (ALMEIDA e FREIRE, 2006; ALMEIDA e RIBEIRO, 2009), por exemplo, as áreas de manguezais foram severamente reduzidas em prol do desenvolvimento econômico com a anuência dos órgãos públicos responsáveis pela sua preservação.

Em Sergipe, particularmente em sua capital, extensas áreas de manguezais cederam lugar para infraestrutura urbana, viária e industrial. Em Aracaju, é notória a preocupação com a degradação dos manguezais causada pela ocupação irregular de suas áreas, ação realizada principalmente por populações de baixa renda. No entanto, isto representa uma pequena parcela de toda a degradação ambiental decorrente do processo de urbanização do município.

É importante observar que a regularização e urbanização de áreas já invadidas, legitimando o processo de ocupação, bem como a criação de diversos novos bairros e estruturas urbanas

$\begin{array}{lllll}\text { Caminhos de Geografia } & \text { Uberlândia } & \text { v. 17, n. } 57 & \text { Mar/2016 } & \text { p. 126-134 Página } 127\end{array}$


foram os maiores responsáveis pela degradação dos manguezais em Sergipe, pois causaram a supressão de grandes faixas de mangue. Estas ações, contaram com a participação do poder público, como é o caso da criação dos bairros Jardins e Coroa do Meio, e da Zona de Expansão de Aracaju, áreas nobres da capital (COSTA et al., 2012; MACHADO, 2010).

Deve-se ressaltar que estas ações foram de encontro ao código florestal vigente à época, pois não se enquadravam nas exceções contempladas no mesmo para a ocupação de APP por se tratar de áreas habitacionais e comerciais destinadas a populações de renda média e alta (BRASIL, 1965). Soma-se a isto a supressão de mangues para implantação de empreendimentos de aquicultura, instalados e em operação ao longo dos estuários de todo 0 estado sem observar ao devido processo de licenciamento.

Apesar deste contexto histórico, a Administração Estadual do Meio Ambiente de Sergipe (ADEMA), autarquia especial da administração direta responsável pela execução das ações de regulação e controle do meio ambiente, definiu como uma de suas metas o mapeamento e quantificação dos remanescentes de manguezais em território sergipano como forma de obter as informações necessárias para o planejamento e monitoramento de ações envolvendo este ecossistema.

Para este fim, foram adotadas ferramentas de sensoriamento remoto e geoprocessamento, as quais já demonstraram sua eficácia no monitoramento e mapeamento de endemias (CORREIA et al., 2004; OLIVEIRA et al., 2012), mapeamento de áreas cultivadas (JESUS e EPIPHANIO, 2010; MENKE et al., 2009), modelagem de distribuição de espécies (NABOUT et al., 2009), monitoramento ambiental (BENEDETTI et al., 2013; KAO et al., 2011), e planejamento e gestão do uso de recursos naturais e desenvolvimento urbano (ALMEIDA, 2010; MENKE et al., 2009; SILVA et al., 2006).

Assim, este trabalho teve como objetivos (1) realizar o mapeamento dos manguezais remanescentes em Sergipe, (2) quantificar a área de cada fragmento mapeado, e (3) gerar uma base de dados de acesso público com informações sobre a distribuição deste ecossistema.

\section{MATERIAL E MÉTODOS}

A área estudada foi a região litorânea do estado de Sergipe. Esta corresponde à porção leste do território sergipano, que é recortada por quatro estuários de grandes proporções, sendo eles o do rio São Francisco; rio Sergipe; rio Vaza Barris e do rio Piauí (Figura 1).

O clima predominante nesta área é o tropical quente e úmido, com médias de temperaturas elevadas e alta pluviosidade, concentrada particularmente no período do inverno. Seu relevo apresenta predominância de planícies costeiras, que favorecem o desenvolvimento de florestas de manguezal.

Para a realização deste trabalho foram obtidas imagens de $5.022 \mathrm{Km}^{2}$ do território do estado, divididos em 23 cenas capturadas pela rede de satélites RapidEye. Este valor corresponde a aproximadamente $22,96 \%$ da extensão do estado e abrange 21 dos seus 75 municípios, correspondendo à sua região costeira. As referidas imagens são multiespectrais, compostas por cinco bandas, sendo elas as três do espectro visível, a infravermelho próximo e a red edge, com resolução espacial de 5 metros e espectral 12 bits.

Para tratamento preliminar das imagens foi aplicado a cada uma o Índice de Vegetação por Diferença Normalizada (NDVI), algorítimo gráfico que responde a variações de biomassa, clorofila e estrutura do dossel (HANSEN e SCHJOERRING, 2003; SADER et al., 1989). De cada cena, foram removidas quaisquer áreas com índice NDVI $\leq 0,2$. Esta medida procurou reduzir a geração de falsos positivos na classificação das imagens, excluindo das mesmas os estratos não arbóreos da vegetação, uma vez que o alvo deste trabalho são as florestas de mangue, que apresentam índices acima deste valor.

Para a detecção das áreas de mangue nas imagens resultantes, o modelo de classificação foi treinado com a coleta de amostras de pixels correspondentes a esta fitofisionomia, a partir de coordenadas coletadas em campo para cada cena separadamente. A coleta independente por cena teve como objetivo minimizar quaisquer distorções causadas por variações nas condições espaciais ou temporais das imagens.

$\begin{array}{llllll}\text { Caminhos de Geografia } & \text { Uberlândia } & \text { v. 17, n. } 57 & \text { Mar/2016 } & \text { p. 126-134 Página } 128\end{array}$


Figura 1. Mapa da área de estudos.

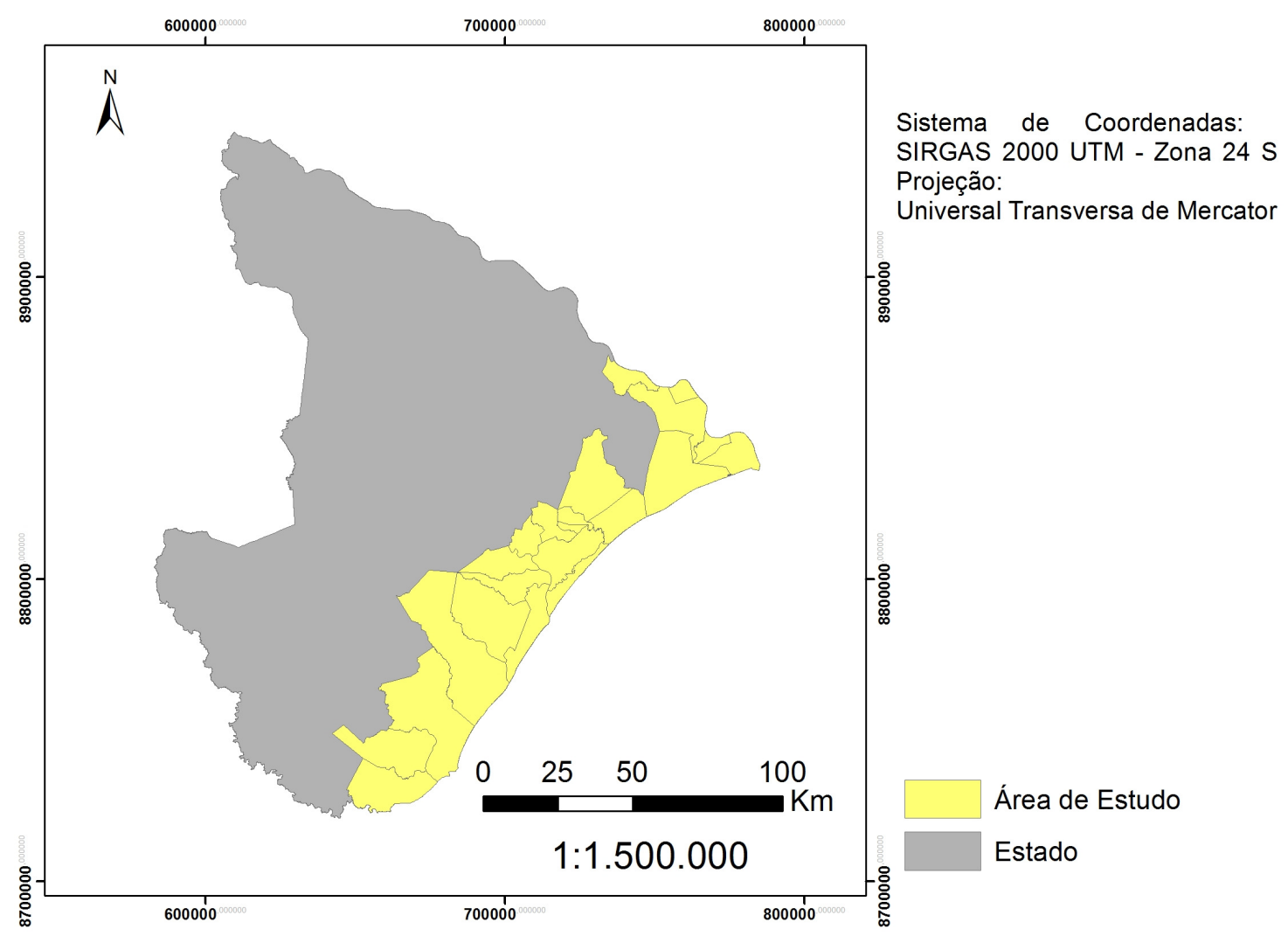

Estas amostras foram introduzidas em um banco de assinaturas do programa, e gerou-se um arquivo contendo a base de informações para a aplicação do algoritmo de busca na imagem. Com base nestes dados, foi realizada a classificação supervisionada da mesma, resultando em um conjunto de mapas temáticos aqui denominados pré-classificados.

Os mapas temáticos pré-classificados foram submetidos à revisão dos técnicos, para levantar possíveis erros de classificação do programa. Expedições técnicas foram realizadas aos pontos de possíveis falsos positivos para a consolidação dos dados. Além disso, foram usadas imagens de bases públicas, como o Google Earth, para validação dos resultados. Ao final do processo de validação as correções foram realizadas manualmente a partir dos dados obtidos em campo e virtualmente.

Após a consolidação das informações obtidas, foi gerado um mosaico dos mapas temáticos referentes a cada imagem, resultando em um único arquivo shape contendo as feições de manguezal do estado.

Para a divulgação destes dados foram utilizados a compilação de um DVD e a publicação de bancos de dados virtuais das informações geradas por este trabalho.

\section{RESULTADOS E DISCUSSÃO}

Neste trabalho foram mapeados e delimitados 251,0786 Km² de mangues, distribuídos em 16 municípios sergipanos. Este valor corresponde a aproximadamente $1,14 \%$ do território do Estado, e se distribui em quatro áreas principais, localizadas nos estuários e ao longo dos principais afluentes dos rios Piauí, Vaza Barris, Sergipe e São Francisco (Figuras 2 e 3).

A metodologia aplicada gerou a classificação de $577,2288 \mathrm{Km}^{2}$ de vegetação, sendo que destes, 210,9433 $\mathrm{Km}^{2}$ (36,54\%) foram confirmados como manguezais, e 366,2855 $\mathrm{Km}^{2}$ $(63,46 \%)$ pertencentes a outros ecossistemas. Os cerca de 40,1353 $\mathrm{Km}^{2}$ restantes corresponderam majoritariamente a áreas de borda ou núcleos não classificados de maneira adequada principalmente por sombreamento de nuvens ou dossel menos denso.

$\begin{array}{llllll}\text { Caminhos de Geografia } & \text { Uberlândia } & \text { v. 17, n. } 57 & \text { Mar/2016 } & \text { p. 126-134 Página } 129\end{array}$


Figura 2. Mapa Temático dos Manguezais de Sergipe.

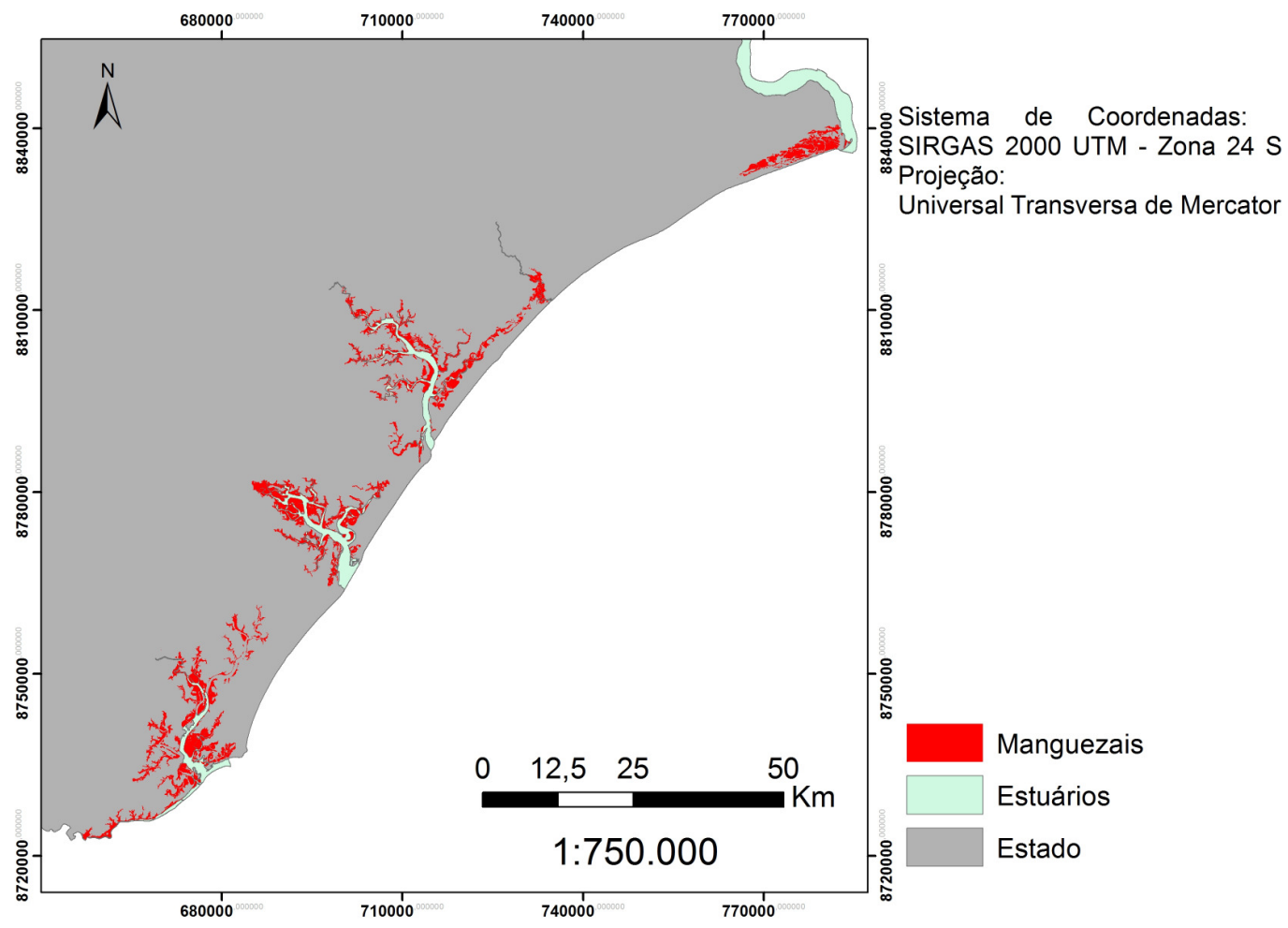

Figura 3. Mapa temático dos manguezais do estuário do rio São Francisco, Sergipe.

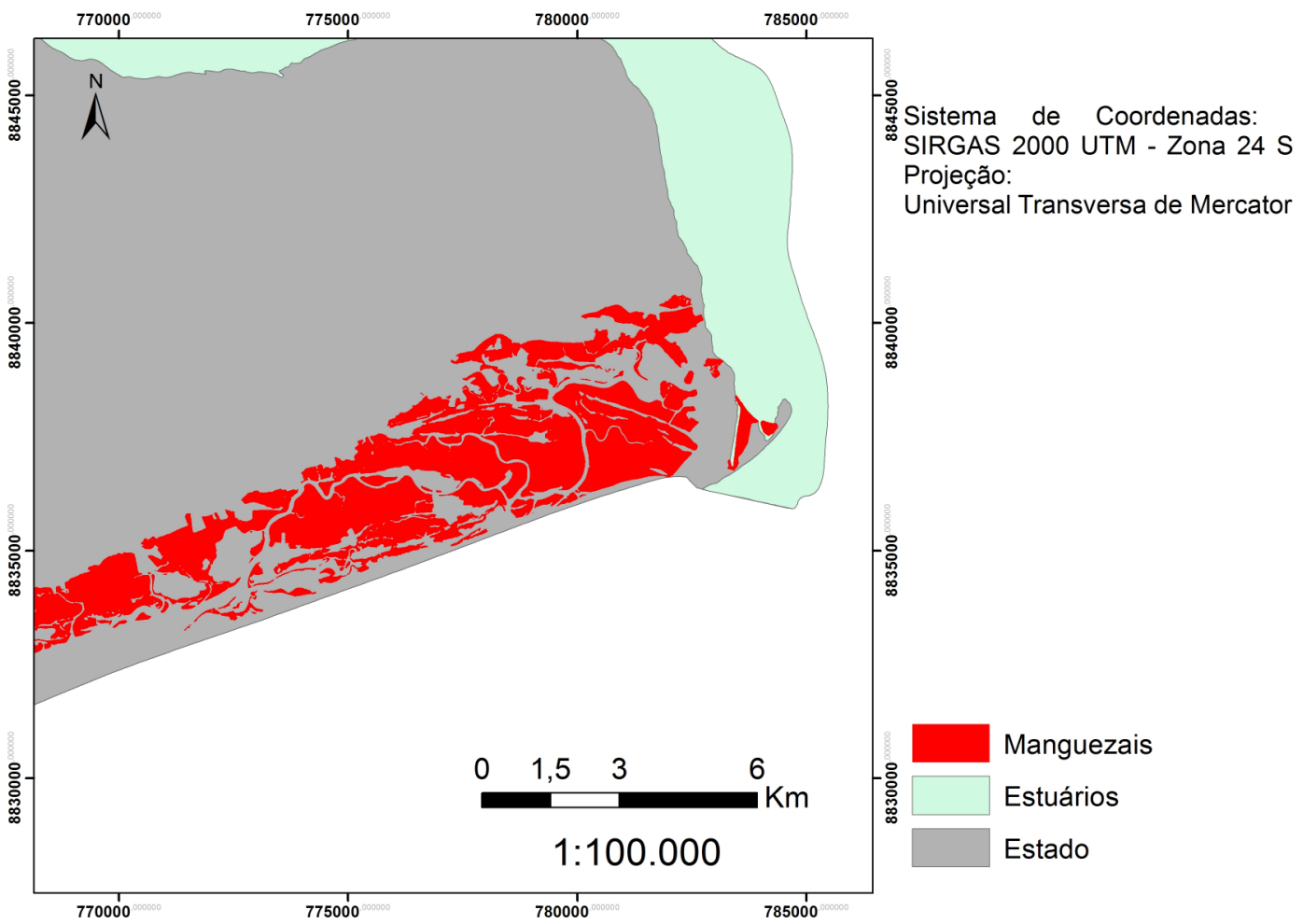

Apesar da elevada margem de erros, no que diz respeito a diferenciação entre manguezais e floresta atlântica stricto sensu, a resposta da classificação para a identificação das áreas de 
manguezais foi bastante significativa. A metodologia classificou automaticamente $84,01 \%$ da área total de manguezais corretamente. No entanto, a maior barreira para a completa automação do processo é a classificação errônea de outras fisionomias. Este tipo de erro pode ser minimizado com a adoção de imagens com um maior número de bandas, que apresentem respostas mais relevantes à variação da reflectância foliar.

A similaridade das respostas espectrais das fitofisionomias fica evidente no histograma das amostras obtidas para cada uma. Para as bandas do espectro visível, Banda 1, Azul, Banda 2, Verde e Banda 3, Vermelho, o comportamento é bastante similar (Figura 4), gerando sobreposição das assinaturas espectrais. Problema similar foi encontrado em outros estudos, como a classificação de estágios sucessionais em florestas (AMARAL et al., 2009) e a classificação de áreas úmidas (SHANMUGAM et al., 2006). Isto ocorre quando são aplicados métodos tradicionais de classificação baseada em pixel, principalmente em função da limitada capacidade dos sensores atuais de diferenciar assinaturas espectrais muito próximas (SHANMUGAM et al., 2006).

Figura 4. Perfil espectral das amostras de mata atlântica stricto senso (preto) e manguezal (cinza), para o espectro visível.

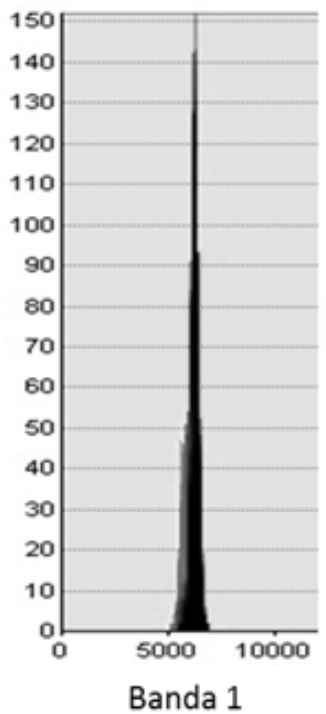

Banda 1

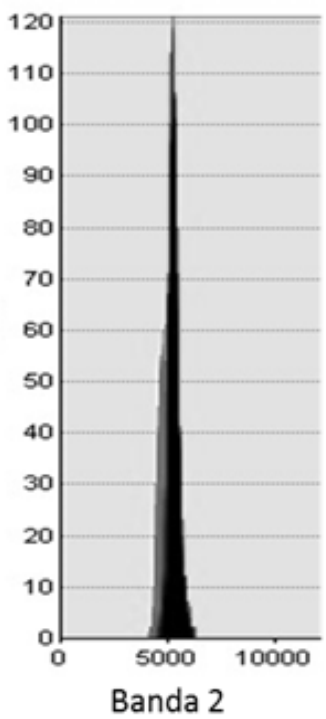

Banda 2

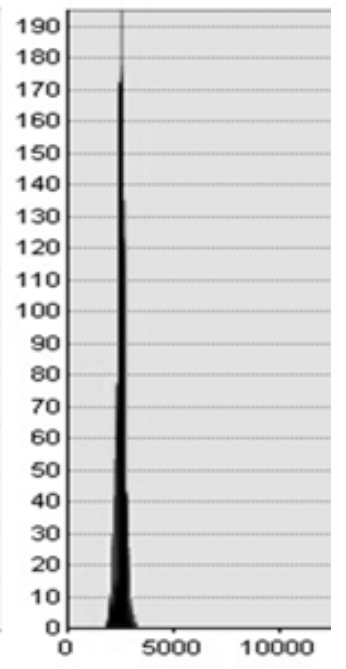

Banda 3

A diferença entre as fisionomias é mais evidente nas bandas termais, sendo que na banda 4, red edge, menos intensamente que na Banda 5, Infravermelho Próximo (Figura 5). Este comportamento é mais provavelmente associado às interferências geradas pela tipologia de solo no qual cada fitofisionomia se desenvolve. Os manguezais estão associados a solos siltosos e argilosos com características hidromórficas, os quais tendem a ter comportamentos térmico bastante distinto do solo seco e predominantemente húmico das áreas de mata atlântica stricto senso.

Acerca da área de remanescentes, levantamentos anteriores realizados pela SOS Mata Atlântica e Instituto Nacional de Pesquisas Espaciais (INPE) (2011) quantificaram as áreas de mata atlântica, em levantamento realizado entre 2008 e 2010, indicando uma área total de $1.098,87 \mathrm{Km}^{2}$, dos quais $229,56 \mathrm{Km}^{2}$ eram áreas de manguezal, uma diferença aproximada de $8,6 \%$ com relação ao total mapeado neste trabalho. A disparidade entre estes trabalhos pode ser explicada pelas divergências metodológicas, pois este último usou fotointerpretação de imagens de satélite Landsat 5 , cuja resolução espacial é mais baixa que aquelas RapidEye (SOS MATA ATLÂNTICA e INPE, 2011).

Considerando ainda os dados existentes, os manguezais representam de 20,89 a $22,84 \%$ de todos os remanescentes de floresta atlântica presentes no estado, se considerados 0 mapeamento do atlas e este estudo respectivamente (SOS MATA ATLÂNTICA e INPE, 2011). Esta situação ilustra a necessidade de sua conservação, vista sua representatividade para 0 bioma no estado. 
Figura 5. Perfil espectral das amostras de mata atlântica stricto senso (preto) e manguezal (cinza) para as bandas térmicas.

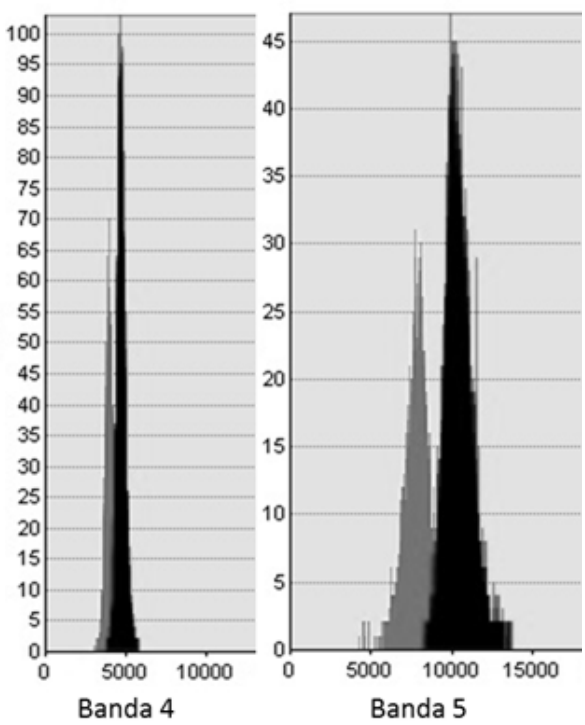

Para que o máximo do potencial deste trabalho pudesse ser extraído, os mapas temáticos resultantes foram convertidos em formato shp (shapefile), e compilados em DVDs, acrescidos de outras informações já disponíveis e relevantes para a interpretação das informações como hidrografia, limites municipais, limites do estado, dentre outras, já disponíveis em base de dados gratuita da Secretaria de Estado do Meio Ambiente e dos Recursos Hídricos (SERGIPE, 2012), e fornecidos também de forma gratuita ao público em geral, mas principalmente para os órgãos municipais e federais de meio ambiente.

A fim de ampliar a abrangência da divulgação, foi estruturado um banco de dados geoespacial, disponível para acesso público e que permite a visualização e download dos dados publicados, com acesso disponível através do site da ADEMA.

\section{CONSIDERAÇÕES FINAIS}

Apesar da possibilidade de maior refino deste trabalho, com o advento de novas tecnologias em um futuro próximo, este mapeamento é um documento importante pois constitui memória para estudos futuros sobre qualquer transformação nos manguezais sergipanos. Além disto, pode subsidiar ações que tenham interface com a sua manutenção no âmbito da administração pública, como o licenciamento ambiental, a fiscalização e o monitoramento destes ambientes, o planejamento urbano, além da pesquisa científica.

Os mapas temáticos gerados como resultado deste trabalho podem ser amplamente aplicados como importante ferramenta para a gestão ambiental. É necessário, contudo, que haja uma estruturação das entidades interessadas, como órgãos de planejamento e gestão do desenvolvimento urbano, industrial e comercial, órgãos voltados à gestão ambiental e institutos de pesquisa, para que possam estar aptas a absorver as informações disponíveis em benefício do andamento de seus trabalhos.

Verificam-se indícios de que parte dos manguezais mapeados se encontra submetidos a forte pressão antrópica, principalmente em função da ocupação humana de seus entornos. As sedes de alguns municípios do estado se desenvolveram de tal modo que é possível verificar a relação entre o crescimento de sua zona urbana e a fragmentação dos mangues. Este fato aponta para a necessidade de novos estudos voltados ao tema, já abordado, mas em constante evolução.

Este trabalho oferece ainda a perspectiva de modelagem de um processo que realize 0 monitoramento ambiental de forma automatizada, lançando as bases para o registro em escala multitemporal do crescimento e redução de áreas de manguezal a partir de variações em seus 
índices de biomassa, com potencial de subsidiar ações melhor fundamentadas e mais efetivas para a conservação destes ambientes.

\section{AGRADECIMENTOS}

Agradecemos aos técnicos da Santiago e Sintra Consultoria e da ADEMA envolvidos neste processo, que se dedicaram de maneira ímpar para a construção deste produto.

\section{REFERÊNCIAS}

ALMEIDA, C.M. Aplicação dos sistemas de sensoriamento remoto por imagens e o planejamento urbano regional. Arq. Urb., v. 3, p. 98-123, 2010.

ALMEIDA, F.C.; FREIRE, E.O.L. Entre a Terra, o Rio e o Mar: A Tradição de Aterros dos Ecossistemas de Manguezais Aracajuanos-Sergipe (1855-1923). In: III ENCONTRO DA ANPPAS. Anais... Brasília, 2006. Disponível em: <http://www.anppas.org.br/ encontro_anual/encontro3/arquivos/TA358-04042006-93201.DOC>. Acesso em: 20 de junho de 2014.

ALMEIDA, F.C.; RIBEIRO, A.S. A esfera pública e a devastação dos manguezais aracajuanos, Sergipe, Brasil. REDE, v. 3, n. 1, p. 23-41, 2009.

ALONGI, D.M. Present state and future of the world's mangrove forests. Environmental Conservation, v. 29, n. 03, p. 331-349, 2002.

AMARAL, M. V. F. et al. Assessment and comparison of methods of classification of orbital images for the mapping of forest succession stadiums. Revista Árvore, v. 33, n. 3, p. 575-582, 2009.

BENEDETTI, A.C.P. et al. Product MOD13Q1 Modis sensor for temporal analysis of forests in the Serras do Sudeste and Campanha Meridional of Rio Grande do Sul. Revista Árvore, v. 37, n. 3, p. 459-467, 2013.

BRASIL. Lei 4.771, de 15 de setembro de 1965. Disponível em: <http://www.planalto.gov .br/ccivil_03/Leis/L4771.htm>. Acesso em: 20 de junho de 2014.1965.

Resolução CONAMA n. 369, de 28 de março de 2006. Diário Oficial da União, Brasília, 28 de março de 2006.

CORREIA, V.R.M. et al. Remote sensing as a tool to survey endemic diseases in Brazil. Cadernos de Saúde Pública, v. 20, n. 4, p. 891-904, 2004.

COSTA, C.S. et al. As transformações socioambientais e os novos usos na maré do apicum no bairro Coroa do Meio em Aracaju. In: I Seminário Nacional do Grupo de Geoecologia e Planejamento Territorial e IV Seminário do GEOPLAN. Anais... Sergipe, 2012.

DUKE, N.C. et al. A world without mangroves? Science, v. 317, n. 5834, p. 41-42, 2007.

FIDELMAN, P.I. et al. Aspectos legais da proteção do ecossistema manguezal e a realidade no município de Ilhéus. In. XIII Semana Nacional de Oceanografia. Anais... Itajaí: Univali, 2000. p. 9-11.

GILMAN, E. et al. Pacific Island mangroves in a changing climate and rising sea: UNEP Regional Seas Reports and Studies N. 179. United Nations Environment Programme. Regional Seas Programme, Nairobi. 2006.

GUIMARÃES, A.S. et al. Impact of aquaculture on mangrove areas in the northern Pernambuco Coast (Brazil) using remote sensing and geographic information system: Aquaculture and mangrove in Pernambuco (Brazil). Aquaculture Research, v. 41, n. 6, p. 828-838, 2009.

HANSEN, P.M.; SCHJOERRING, J.K. Reflectance measurement of canopy biomass and nitrogen status in wheat crops using normalized difference vegetation indices and partial least squares regression. Remote Sensing of Environment, v. 86, n. 4, p. 542-553, 2003.

JESUS, S.; EPIPHANIO, J.C.N. Sensoriamento remoto multissensores para a avaliação temporal da expansão agrícola municipal. Bragantia, v. 69, n. 4, p. 945-956, 2010.

$\mathrm{KAO}$, P. et al. Decreasing of Population Size of Imperata cylindrica Mangrove Ecotype and Sea-Level Rising. INTECH Open Access Publisher, 2011.

$\begin{array}{llllll}\text { Caminhos de Geografia } & \text { Uberlândia } & \text { v. 17, n. } 57 & \text { Mar/2016 } & \text { p. 126-134 Página } 133\end{array}$


MACHADO, A.B. O bairro Jardins: processo de enobrecimento urbano, consolidação de estratos socioeconômicos e "ilhas" de segregação social. Scientia Plena, v. 6, n. 8, 2010.

MENKE, A.B. et al. Análise das mudanças do uso agrícola da terra a partir de dados de sensoriamento remoto multitemporal no Município de Luis Eduardo Magalhães (BA-Brasil). Sociedade e Natureza, v. 21, n. 3, p. 315-326, 2009.

NABOUT, J. C. et al. Distribuição geográfica potencial de espécies americanas do caranguejo "violinista" (Uca spp.) (Crustacea, Decapoda) com base em modelagem de nicho ecológico. Iheringia, Série Zoologia [online], v. 99, p. 92-98, 2009.

NOGUEIRA, R.X.S.; DOMINGUEZ, J.M.L. Change detection at Conde municipality coastal zone, State of Bahia, Brazil-using medium resolution satellite data. Journal of Coastal Research, v. 50, p. 33-37, 2007.

OLIVEIRA, E.F. et al. Biotic factors and occurrence of Lutzomyia longipalpis in endemic area of visceral leishmaniasis, Mato Grosso do Sul, Brazil. Memórias do Instituto Oswaldo Cruz, v. 107, n. 3, p. 396-401, 2012.

RODRIGUES, F.O. et al. Environmental impact in mangrove ecosystems: São Paulo, Brazil. In: YÁNEZ-ARANCIBIA, A.; LARA-DOMÍNGUEZ, A. L. (Eds.). Ecosistemas de manglar en América Tropical. México: Instituto de Ecología A.C.; San Jose: UICN/ORMA; Silver Spring: NOAA/NMFS, 1999. p. 175-198.

RÖNNBÄCK, P. The ecological basis for economic value of seafood production supported by mangrove ecosystems. Ecological Economics, v. 29, n. 2, p. 235-252, 1999.

SADER, S.A. et al. Tropical forest biomass and successional age class relationships to a vegetation index derived from Landsat TM data. Remote Sensing of Environment, v. 28, p. 143-198, 1989.

SERGIPE. Atlas Digital Sobre Recursos Hídricos. Sergipe: Secretaria de Estado do Meio Ambiente e Recursos Hídricos, 2012.

SHANMUGAM, P. et al. Comparison of the classification of wetland characteristics by linear spectral mixture modelling and traditional hard classifiers on multispectral remotely sensed imagery in southern India. Ecological Modelling, v. 194, n. 4, p. 379-394, 2006.

SILVA, H.R. et al. Sensoriamento remoto na identificação do uso e ocupação da terra na área do projeto" Conquista da Água", Ilha Solteira-SP, Brasil. Engenharia Agrícola, v. 26, n. 1, p. 328-334, 2006.

SOS MATA ATLÂNTICA; INPE - Instituto Nacional de Pesquisas Espaciais. Atlas dos remanescentes florestais da Mata Atlântica período 2008-2010. Fundação SOS Mata Atlântica, São Paulo, 2010.

VALIELA, I. et al. Mangrove Forests: One of the World's Threatened Major Tropical Environments At least 35\% of the area of mangrove forests has been lost in the past two decades, losses that exceed those for tropical rain forests and coral reefs, two other well-known threatened environments. Bioscience, v. 51, n. 10, p. 807-815, 2001.

WILKIE, M.L.; FORTUNA, S. Status and trends in mangrove area extent worldwide. Forest Resources Assessment Programme. Forest Resources Division, FAO, Paris 2003. 